\title{
A case from the Nottingham Breast Institute: An unusual cause of microcalcifications detected at breast cancer screening
}

\author{
J. J. James \\ Nottingham Breast Institute, Nottingham City Hospital, Hucknall Road, Nottingham, UK.
}

\section{Introduction}

Detecting microcalcifications in the breast with mammographic screening is of great importance. Ductal carcinoma in situ (DCIS) is commonly detected as foci of breast microcalcifications. The problem is that many benign changes in the breast parenchyma and the skin also contain microcalcifications.

Many women are recalled for assessment of screen-detected microcalcifications. Additional mammographic views, clinical examination and biopsy are all used to differentiate benign from malignant disease. This case describes an unusual cause of screen-detected 'microcalcifications'.

\section{Case report}

A 49-year-old Caucasian female was invited to attend for her first screening mammogram. A 35-mmdiameter curvilinear cluster of microcalcifications was demonstrated on both the mediolateral oblique projection (Fig. 1(a) and (b)) and the craniocaudal projection in the 12 o'clock position in the left breast. The woman was recalled for further assessment.

At the assessment clinic it was noted that the microcalcifications corresponded to a large strawberry tattooed on the patient's left breast (Fig. 1(c)). Lead shot was placed on the patient's skin and an oblique film repeated to ensure that the calcifications did correspond to the tattoo. The curvilinear configuration of the calcifications appeared to represent

Correspondence to: J. J. James, FRCR, Consultant Radiologist, Nottingham Breast Institute, Nottingham City Hospital, Hucknall Road, Nottingham, UK. E-mail: jjames3@ncht.trent.nhs.uk; Tel: +0 115962 7725; Fax: +0 115 9627707

Publication date 31/03/05 BCO $/ 407 / 2005 / C S$

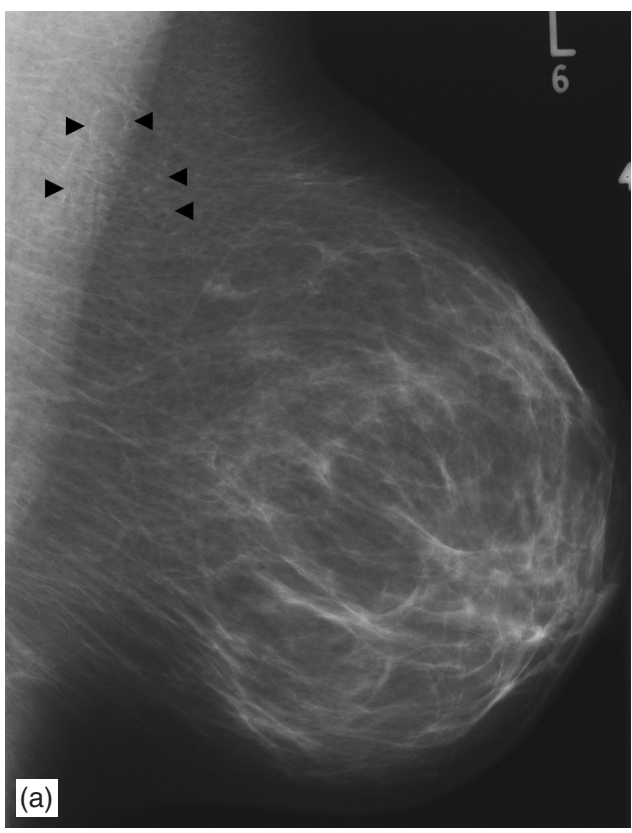

Figure 1(a).

Mediolateral oblique mammogram (a) of the left breast showing clusters of 'microcalcifications' partially overlying the pectoral muscle (arrowheads).

the green leaves of the strawberry. The tattoo had been obtained 2 years previously.

The women was reassured regarding the benign nature of the microcalcifications and returned to routine screening.

\section{Discussion}

Tattooing has been practised by many cultures from ancient times. In Western society its popularity fluctuates, but in recent years its use as a form of 'body art' has seen something of a renaissance. Its use these 

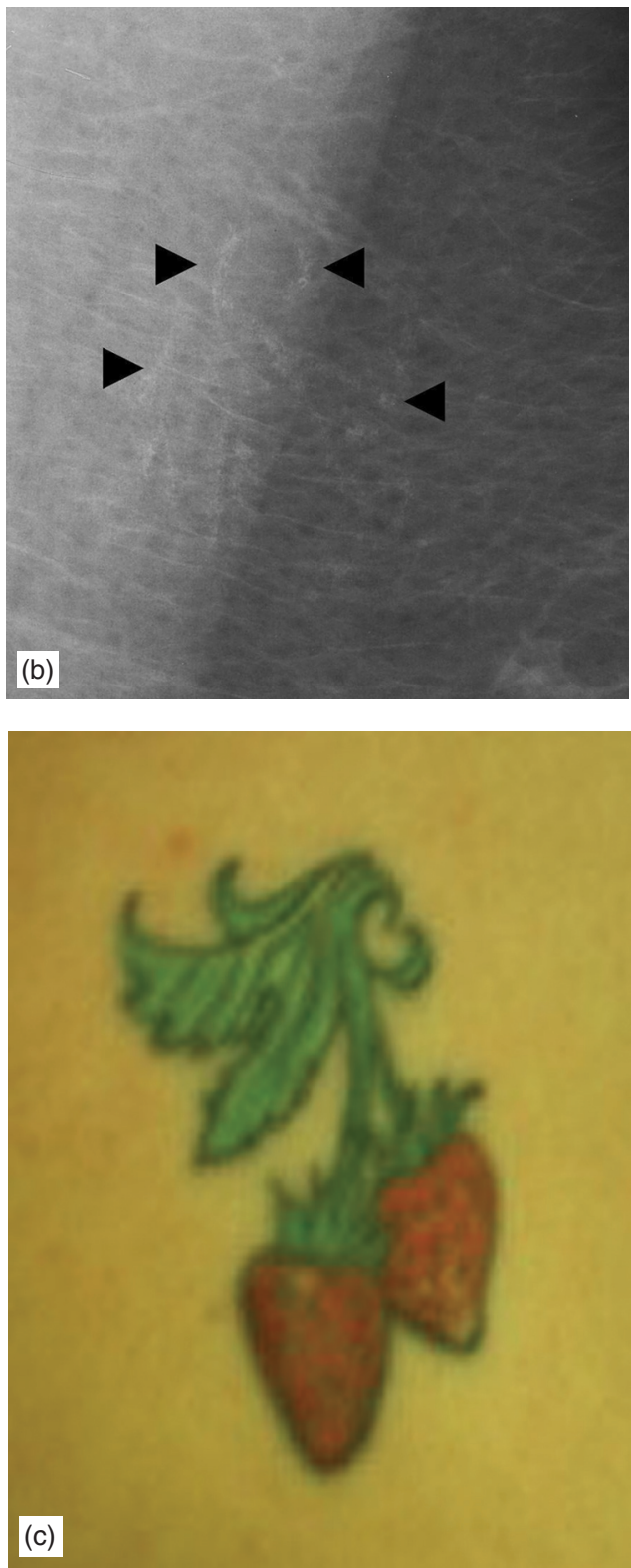

Figure 1(b, c).

The curvilinear layout of the microcalcifications is better appreciated on the magnification view (b) (arrowheads). The microcalcifications correspond with the green leaves and stems of the strawberry tattoo (c).

days is not exclusive to the young or to any particular social group. No part of the body seems immune to the professional tattooists needle, even the breast. An electric needle is used to introduce coloured pigments into the dermis of the skin. Many of these pigments contain metal salts and other radio-opaque particles that are potentially visible on a mammogram.

To my knowledge there has only been one reported similar case in the literature about 20 years ago [1]. A similar problem has been reported when tattoo pigments were used to orientate excised surgical breast tissue prior to specimen radiography [2]. There has also been a report of tattoo pigments migrating to lymph nodes mimicking calcifications in an axillary node [3]. In this case, it appears to be the green pigment in the strawberry leaves that was visible on the mammogram. The composition of elements in tattoo inks varies greatly even among like-coloured compounds. The most commonly identified metallic elements in tattoo ink are aluminium (87\% of pigments) and titanium (67\% of pigments) [4].

It is well recognised that benign calcifications occur naturally in the subcutaneous tissues of the breast, usually within sebaceous glands. These may mimic suspicious breast parenchymal microcalcifications on the screening mammogram. Artefacts such as powder or ointment on the patient's skin may cause similar confusion. Sometimes repeat mammograms and tangential views are all that is required for clarification.

Other skin lesions such as moles, papillomas and scars are all potential causes of mammographic abnormalities. The examining radiographer usually notes the presence of such lesions at the time of mammography to aid interpretation of the screening mammogram. Patient recall to the assessment clinic may have been avoided, if the radiographer had noted the presence and location of the tattoo. It is always essential to be sure that such skin lesions are the cause of the abnormality and if any doubt exists then further assessment is required. Repeat films after placing lead shot on the lesion is a reliable way of confirming the correlation of the mammographic abnormality to the skin lesion. The same is equally true for tattoos.

A tattoo remains a rare cause of apparent microcalcifications on the screening mammogram. With the growing popularity of professional tattooing with coloured pigments, many screening units may encounter similar cases in coming years.

\section{References}

1. Brown RC, Zuehlke RL, Ehrhardt JC, Jochimsen PR. Tattoos simulating calcifications on xeroradiographs of the breast. Radiology 1981; 138: 583-584.

2. Lager DJ, O'Connor JC, Robinson RA, Brown RC, Urdaneta LF. Factitious microcalcifications in breast biopsy material: laboratory induced error by use of tattoo powder for specimen mammography. J Surg Oncol 1989; 40: 281-282.

3. Honegger MM, Hesseltine SM, Gross JD, Singer C, Cohen JM. Tattoo pigment mimicking axillary lymph node calcifications on mammography. Am J Radiol 2004; 183: 831-832.

4. Timko AL, Miller CH, Johnson FB, Ross V. In vitro quantitative chemical analysis of tattoo pigments. Arch Dermatol $2001 ; 137: 143-147$. 